\title{
HERRAMIENTAS DE ECONOMÍA ECOLÓGICA PARA LA EVALUACIÓN DE LA SOSTENIBILIDAD DE BIOMASAS
}

GREEN ECONOMIC TOOLS FOR THE ASSESSMENT OF SUSTAINABILITY OF BIOMASSES

Reinier Jiménez Borges*

https://orcid.org/0000-0001-6451-8499

Andrés Lorenzo Álvarez González**

https://orcid.org/0000-0002-3302-1540

Eduardo Julio López Bastida ${ }^{\dagger}$

https://orcid.org/0000-0003-1305-852X

Aliena de la Caridad Bermúdez Chou ${ }^{\ddagger}$

https://orcid.org/0000-0003-0581-0283

UNIVERSIDAD DE CIENFUEGOS CARLOS RAFAEL RODRÍGUEZ

Recibido: 3 de diciembre de 2019

Aceptado: 5 de febrero de 2020

DOI: https://doi.org/10.29097/2011-639X.276

\section{Resumen}

En el presente estudio se aplicaron algunas de las herramientas de la economía ecológica para evaluar la sostenibilidad de las principales biomasas en la provincia de Cienfuegos, Cuba. Para ello se seleccionaron como principales biomasas el bagazo de caña; cachaza, como residuo del proceso de clarificación del jugo de caña; los llamados "Residuos Agrícolas de Cosecha” (RAC), cascarilla de arroz y residuos forestales. Se determinaron las cantidades disponibles a través del levantamiento en cada unidad productora para, con ello, estimar la energía total y la reducción de $\mathrm{CO}_{2}$ arrojada a la atmósfera para cada fuente de biomasas. Finalmente, se aplicó el método de jerarquía analítica (AHP en inglés) como técnica de decisión de multicriterios para evaluar la sostenibilidad de las alternativas de biomasas presentadas. Además, se realizó un análisis de sensibilidad debido a la subjetividad que presenta esta técnica de decisión.

Palabras clave: biomasa, economía ecológica, indicadores, sostenibilidad, Método de Jerarquía Analítica (AHP).

\section{Abstract}

This study applied some of the tools of ecological economics to assess the sustainability of the main biomasses in Cienfuegos province, Cuba. For this purpose, the following were selected as the main biomasses: bagasse; cachaza or press cake, as a by-product of the sugarcane juice clarification process, Agricultural Harvest Waste (AHR), rice husks

* Magíster en Eficiencia Energética. Profesor asistente del Centro de Estudios de Energía y Medio Ambiente (CEEMA). Facultad de Ingeniería. Universidad de Cienfuegos. $₫$ rjborges@ucf.edu.cu

** Ingeniero mecánico. Departamento de Mecánica. Universidad de Cienfuegos.

† Doctor en Ciencias Técnicas. Profesor titular. Centro de Estudios de Energía y Medio Ambiente (CEEMA). Facultad de Ingeniería. Universidad de Cienfuegos.

‡ Estudiante de 4to año de Ingeniería Mecánica. Facultad de Ingeniería. Universidad de Cienfuegos. 
and forest residues. The quantities available were determined through the survey in each production unit, in order to estimate the total energy, as well as the reduction of $\mathrm{CO}_{2}$ released into the atmosphere for each biomass source. Finally, the Analytical Hierarchy Process (AHP) was applied as a multi-criteria decision technique to evaluate the sustainability of the biomass alternatives presented. In addition, a sensitivity analysis was carried out, due to the subjectivity of this decision technique.

Keywords: biomass, ecological economics, indicators, sustainability, Analytical Hierarchy (AHP).

\section{INTRODUCCIÓN}

En los últimos veinte años se han desarrollado tecnologías que posibilitan dar saltos importantes en la eficiencia, basadas en combustibles renovables como el bagazo y la paja de caña. Hoy existen instalaciones capaces de elevar la eficiencia energética desde un 10 a un $15 \%$ más. Al mismo tiempo se desarrollan otras tecnologías más avanzadas, como las turbinas de gas integradas con gasificadores de biomasa, que podrían entonces elevar los valores entre un 20 y 30 \% (Terence James, Beltrán Fundora \& Sendon Medina, 2010).

Esos avances tecnológicos hacen competitiva la generación de electricidad a base de biomasa, si se compara con la obtenida de combustibles fósiles. La combustión de la biomasa tiene, además, una ventaja ambiental, ya que disminuye la concentración atmosférica de carbono, porque solo devuelve a la atmósfera el carbono que fijó la planta durante su crecimiento (Cerdá, 2012; Giuntoli et al., 2016) (Vergara Tamayo \& Ortiz Motta, 2016).

En Cuba, la principal fuente de energía renovable es la biomasa, y el mayor potencial energético lo tiene la biomasa cañera (Hernández, 2018). Actualmente es baja la utilización de las fuentes renovables de energía, pues con ellas solo se produce el 4,3 \% de la electricidad del país donde la biomasa alcanza el 3,5 \% (Guerra, 2016). La provincia de Cienfuegos se localiza en la zona central de Cuba y ocupa $4.178 \mathrm{~km}^{2}$ (417.800 ha.). Presenta una economía variada basada en la agricultura, industria y el turismo (ONEI, 2019). En Cuba, la Oficina Nacional de Estadística e Información (ONEI) ofrece estadística generalizada de la situación de la biomasa en las provincias, aunque de forma inespecífica, por lo que se desconoce el potencial energético que puede aportar cada una de ellas. Es imprescindible, entonces, contar con un levantamiento de datos certero sobre la disponibilidad actual de las biomasas a través de cada unidad productora.

En este estudio se consideraron las biomasas de la industria azucarera, arrocera y los residuos agroforestales, con los datos aportados por la empresa azucarera y el grupo agroforestal pertenecientes al territorio (AZCUBA, 2019) (GAF, 2019). Por otro lado, los estudios actuales se centran en determinar los aportes energéticos (Ozoegwua, 2017), (Jiménez Borges, 2017), (Suzuki, 2017), (Ozturka, 2017), (Ozcan, 2017), (Toklu, 2017), (Li, 2017), (Jiang, 2017), (Burg, 2018), (Zhong, 2018), (Rahayu, 2018), mientras que otros determinan indicadores medioambientales como las reducciones de $\mathrm{CO}_{2}$. De allí 
se hace necesaria la realización de estudios sobre herramientas o indicadores de economía ecológica (Huella Ecológica, Análisis de Ciclo de Vida [ACV], Análisis Multicriterios para la toma de decisiones [MCDA en inglés]), etc., que permitan evaluar la sostenibilidad de la biomasa con un marco integral de aprovechamiento. Los MCDA son ampliamente utilizados en los estudios sobre planeación energética que abarcan fuentes renovables de energía (Aras, Erdogmus \& Koc, 2004), (Lee, Chen \& Kang, 2009) (Amer \& Daim, 2011), (Nixon, Dey \& Ghosh, 2013), (Ahmad \& Tahar, 2014), (Al Garni, Kassem, Awasthi, Komljenovic \& Al-Haddad, 2016). Para el caso particular se decidió complementar el análisis energético con la determinación de la cantidad de $\mathrm{CO}_{2}$, dejada de emitir a la atmósfera, y el análisis multicriterio para la toma de decisiones; este como objetivo fundamental del estudio.

\section{METODOLOGÍA}

Como complemento al diseño de la metodología (ver figura 1), se realiza la valoración desde la economía ecológica (EE) mediante la obtención de indicadores de sostenibilidad como la reducción de $\mathrm{CO}_{2}$ y el método MCDA.

\section{Figura 1.}

Metodología con enfoque de economía ecológica.

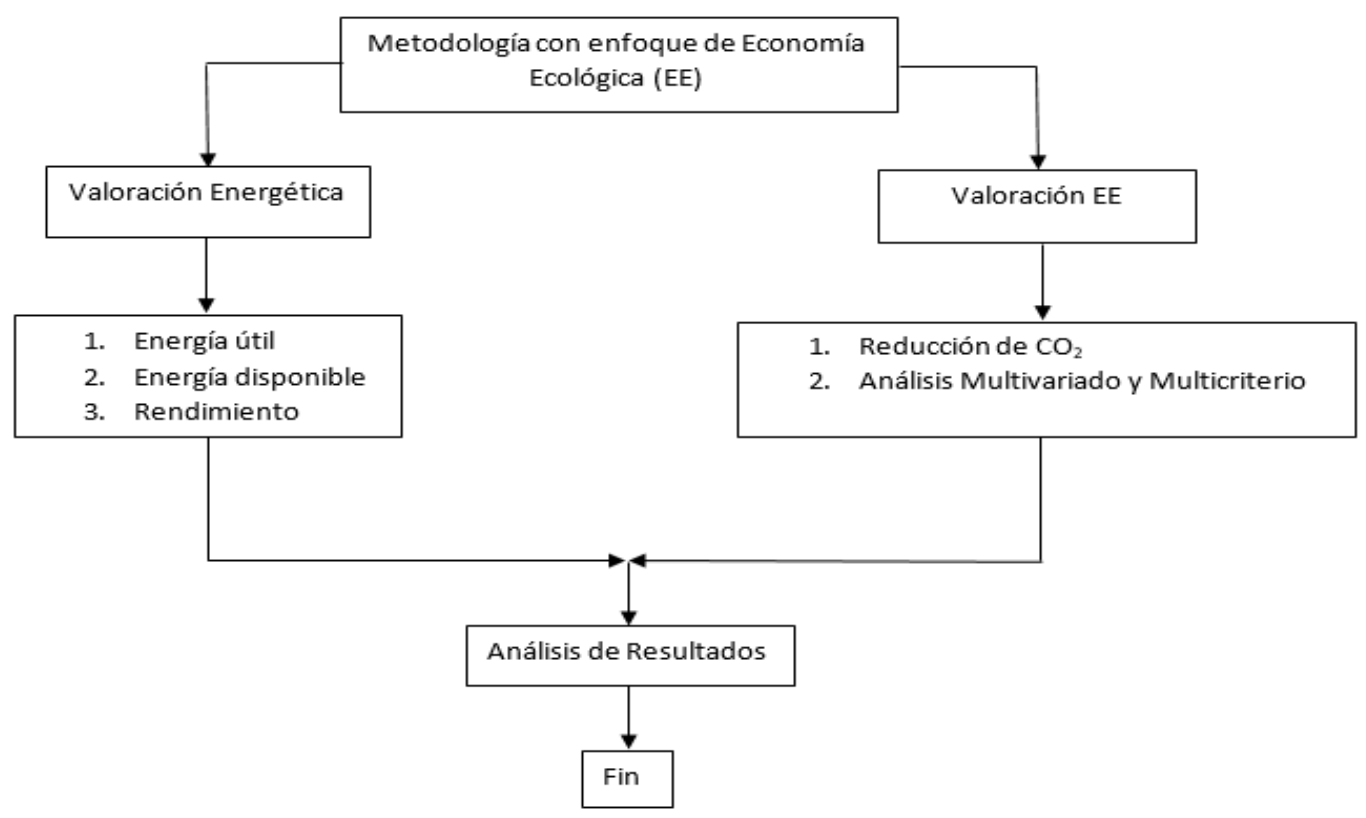

Nota: La figura (de elaboración propia) presenta la metodología propuesta con un enfoque de economía ecológica, que comienza con la determinación de las potencialidades energéticas derivadas de biomasas a través de una valoración de su energía útil, disponible y de su rendimiento. 
Jiménez Borges, Álvarez González, López Bastida y Bermúdez Chou

Tabla 1

Composición química y valores calóricos para las distintas fuentes de biomasas

\begin{tabular}{|c|c|c|c|c|c|c|c|}
\hline Biomasas & $\mathbf{H R ( \% )}$ & $\mathbf{C ( \% )}$ & $\mathbf{H}(\%)$ & $\mathbf{O ( \% )}$ & Cenizas(\%) & $\begin{array}{c}\mathbf{( P C I} \text {.. }) \\
(\mathbf{M J} / \mathbf{k g})\end{array}$ & $\begin{array}{c}\left.\mathbf{P C S}_{\text {b.s }}\right) \\
\mathbf{( M J} / \mathbf{k g})\end{array}$ \\
\hline Bagazo de caña & 50 & 47,2 & 7,0 & 43,1 & 2,7 & 15,8 & 17,3 \\
\hline Cachaza & $70-80$ & 32,5 & 2,2 & 2,2 & 14,5 & 8,8 & 14,5 \\
\hline Paja de caña & 45 & 43,5 & 6,1 & 41,1 & 9,3 & 15,7 & 17,2 \\
\hline Cáscara de arroz & $8-10$ & 38,2 & 5,6 & 33,7 & 22,5 & 15,2 & 16,5 \\
\hline $\begin{array}{c}\text { Residuos } \\
\text { aserraderos }\end{array}$ & 15 & 51,4 & 5,7 & 38,7 & 3,8 & 19,2 & 18,5 \\
\hline
\end{tabular}

Nota: La tabla presenta la composición química y los valores calóricos para cada fuente de biomasa en Cuba (base seca).

La energía total por fuente de biomasa puede ser estimada mediante la ecuación 1:

$$
\mathrm{E}_{\text {total }}=\mathrm{B}_{\text {prod }} * \mathrm{PCS}_{\mathrm{b} . \mathrm{s}}
$$

Donde:

$\mathrm{E}_{\text {total }} \rightarrow$ Energía total (MJ)

$\mathrm{B}_{\text {prod }} \rightarrow$ Cantidad de biomasa producida (kg) (ONEI, 2019)

$\mathrm{PCS}_{\mathrm{b} . \mathrm{s}} \rightarrow$ Poder Calórico Superior en base seca (MJ/kg) (Gutiérrez et al., 2016)

La energía utilizada puede ser determinada utilizando la ecuación 2:

$$
\mathrm{E}_{\text {útil }}=\mathrm{B}_{\mathrm{comb}} * \mathrm{PCS}_{\mathrm{b} . \mathrm{s}}
$$

Donde:

$E_{\text {util }} \rightarrow$ Energía utilizada (MJ)

$\mathrm{B}_{\mathrm{comb}} \rightarrow$ Cantidad de biomasa utilizada como combustible (kg)

El rendimiento puede ser calculado mediante la ecuación 3:

$$
\eta=\frac{E_{\text {útil }}}{E_{\text {total }}}
$$


La cantidad de $\mathrm{CO}_{2}$ dejada de emitir a la atmósfera puede ser determinada mediante la ecuación 4:

$$
\mathrm{E}=\mathrm{E}_{\mathrm{a}} * \mathrm{FE}
$$

Donde:

$\mathrm{E} \rightarrow$ Emisiones $\left(\mathrm{tCO}_{2} / \mathrm{año}\right)$

$\mathrm{E}_{\mathrm{a}} \rightarrow$ Energía ahorrada (kWh/año)

$\mathrm{FE} \rightarrow$ Factor de Emisión (0,001127 tCO 2 kWh) (Narváez, 2013; Oscullo \& Haro, 2016; Resolución conjunta de los Ministerios de Industria, 2016).

La energía ahorrada $E_{a}$ se determinó considerando la cantidad de combustible fósil dejada de consumir en una central convencional con relación al aporte energético de la biomasa en cuestión.

Para determinar las prioridades de las alternativas es necesario resolver las matrices de juicio, utilizando la metodología AHP. El vector de prioridad local (PVE o w) para los juicios matriciales se obtiene normalizando los vectores en cada columna de la matriz (dividiendo la magnitud de cada ponderación por el total de la columna), y luego calculando el promedio de las filas de la matriz resultante. Sin embargo, las prioridades globales para cada alternativa se determinan sintetizando las prioridades locales sobre la jerarquía.

\section{Tabla 2.}

Escala de Saaty de nueve puntos para la comparación por pares.

\begin{tabular}{|c|c|}
\hline Valoración numérica & $\begin{array}{c}\text { Juicios verbales de preferencias } \\
\text { entre alternativas } \boldsymbol{i} \text { y alternativas } \boldsymbol{j}\end{array}$ \\
\hline 1 & $i$ es igualmente importante a $\boldsymbol{j}$ \\
\hline 3 & $i$ es ligeramente más importante que $\boldsymbol{j}$ \\
\hline 5 & $i$ es fuertemente más importante que $\boldsymbol{j}$ \\
\hline 7 & $i$ es rotundamente más importante que $\boldsymbol{j}$ \\
\hline 9 & $i$ es extremadamente más importante que $\boldsymbol{j}$ \\
\hline $2,4,6,8$ & Valores intermedios \\
\hline
\end{tabular}

Nota: Para la aplicación del MCDA, los juicios se basan en una comparación estandarizada de la escala de Saaty de nueve niveles, tal como aparece en la tabla. Fuente: Saaty (1998). 


\section{CONTROL DE CONSISTENCIA}

El control de consistencia garantiza que los juicios de comparación por pares sean lo suficientemente consistentes al calcular la relación de consistencia (CR). Para ello, primero se debe calcular el principio de valor propio ( $\lambda$ máx) para cada matriz, usando la ecuación 5:

$$
\mathrm{A} * \mathrm{w}=\lambda_{\text {máx }} * \mathrm{w}
$$

Donde:

$A \rightarrow$ matriz de comparación

$\lambda$ máx $\rightarrow$ principio del valor propio

$w \rightarrow$ vector propio correctamente normalizado (vector de prioridad).

En segundo lugar, se estima el índice de consistencia (CI) para cada matriz con el tamaño " $n$ " de esta, utilizando la ecuación 6:

$$
\mathrm{CI}=\frac{\lambda_{\text {máx }}-\mathrm{n}}{\mathrm{n}-1}
$$

Finalmente se calcula el CR usando la ecuación 7:

$$
\mathrm{CR}=\frac{\mathrm{CI}}{\mathrm{RI}}
$$

Donde $R I$ es el índice aleatorio. El valor de $R I$ se selecciona según la dimensión de la matriz de comparación ( $n$ ).

\section{Tabla 3.}

Valores de índice aleatorio $(R I)$ para diferentes tamaños de matrices

\begin{tabular}{|c|c|c|c|c|c|c|c|c|c|c|}
\hline Tamaño de la matriz (n) & 1 & 2 & 3 & 4 & 5 & 6 & 7 & 8 & 9 & 10 \\
\hline Índice aleatorio & 0 & 0 & 0,58 & 0,9 & 1,12 & 1,24 & 1,32 & 1,41 & 1,45 & 1,49 \\
\hline
\end{tabular}

Nota: La tabla ilustra los diferentes valores de RI para matrices que tienen un orden n de 1 a 10. Fuente: Qazi et al. (2018).

Una de las partes más relevantes del AHP consiste en la estructuración de la jerarquía del problema, etapa en la cual el grupo decisor involucrado debe lograr desglosar el problema en sus componentes importantes. 
Los pasos que se deben seguir para la estructuración del modelo jerárquico son:

- Identificación del problema.

- Definición del objetivo.

- Identificación de criterios.

- Identificación de alternativas.

\section{Figura 2}

Modelo jerárquico para la toma de decisiones con el AHP.

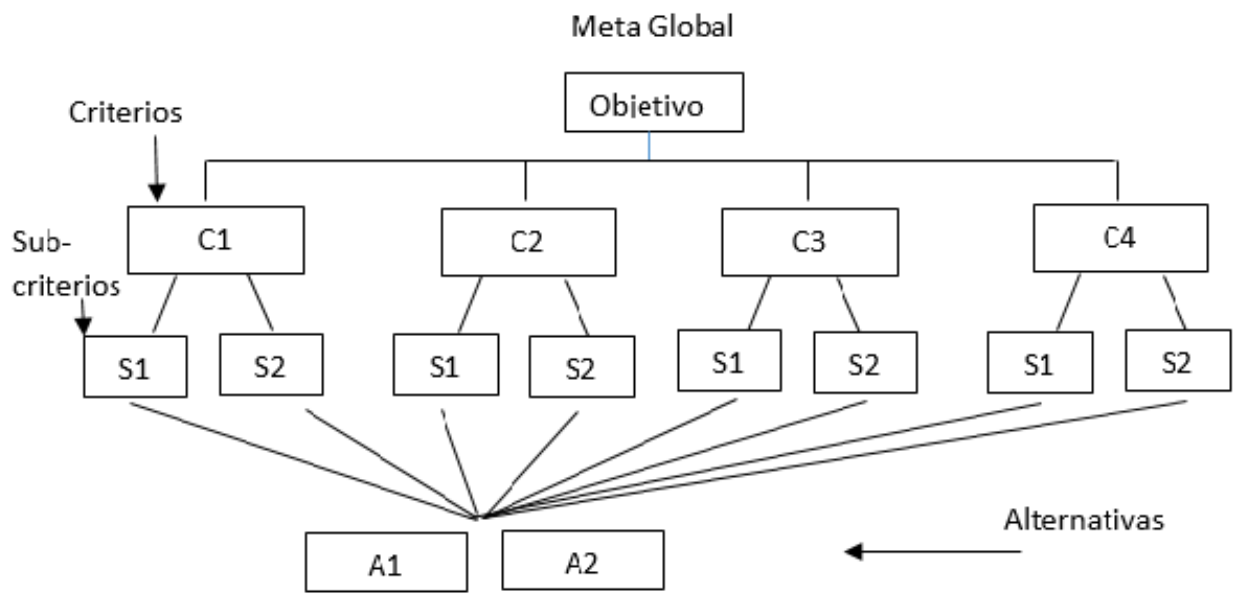

Nota: La jerarquía básica está conformada por: meta u objetivo general, criterios y alternativas. Fuente: (Saaty, 1998).

\section{RESULTADOS}

Para la determinación de la energía total para cada fuente de biomasa es necesario conocer, primero, la composición química de cada una de estas y sus respectivos poderes calóricos. La tabla 1 arriba presenta la composición química y los valores o poderes calóricos para las distintas fuentes de biomasas en Cuba [base seca] (Gutiérrez et al., 2016).

Con el bagazo, como la fuente de biomasa de utilización primordialmente combustible en el territorio, además de su mayor disponibilidad sobrante, se decidió estimar la cantidad de energía utilizada y la eficiencia debido a que presenta la mayor energía total, estimada en 2,45X10 ${ }^{10} \mathrm{MJ}$; utiliza una energía de 2,33X10 ${ }^{10} \mathrm{MJ}$ y tiene un rendimiento del $95 \%$. No obstante, se determinó para las restantes biomasas el potencial energético que aporta cada una. De estas fuentes (ver figura 3), la paja de caña representa alrededor del $60 \%\left(4,02 \times 10^{09} \mathrm{MJ}\right)$ en el aporte de energía, destacándose además la contribución de la cachaza con un 38,79 \% (2,70X10 $\left.{ }^{09}\right)$. 
Jiménez Borges, Álvarez González, López Bastida y Bermúdez Chou

Figura 3.

Potencial energético por fuentes de biomasa en el territorio (MJ).

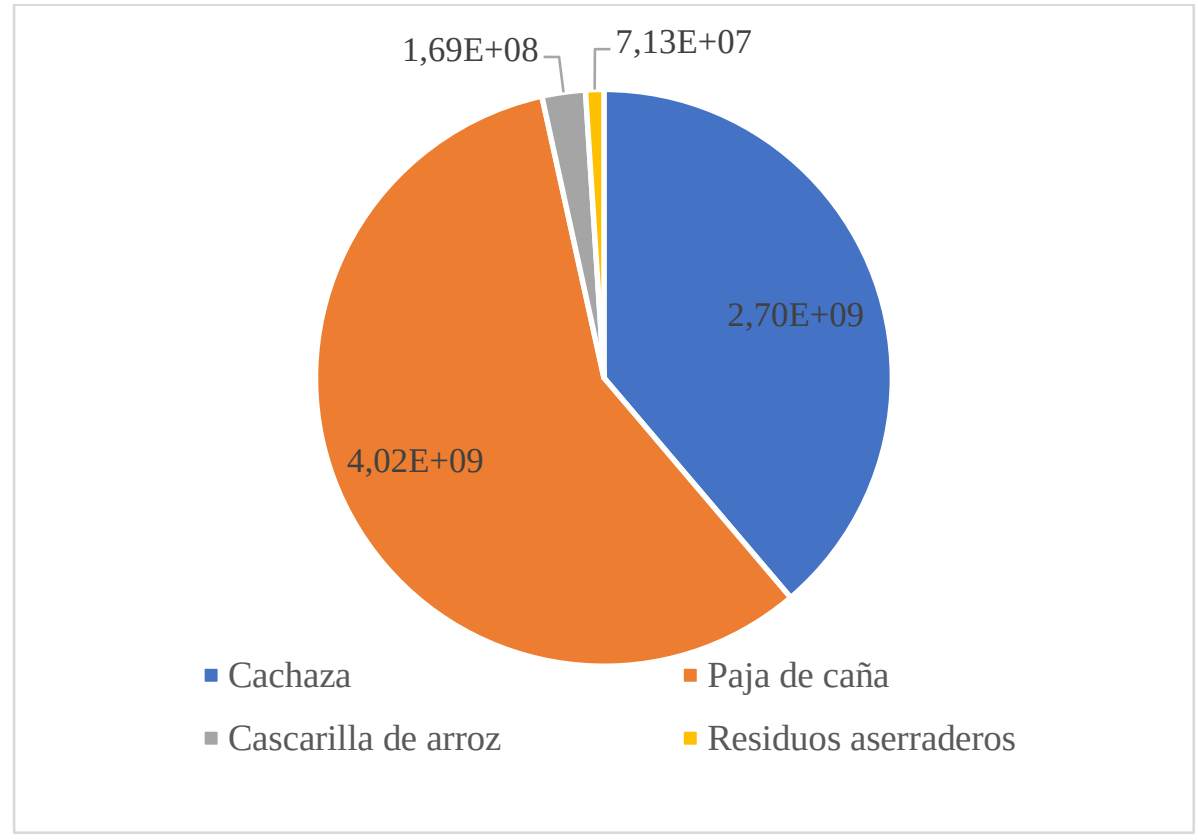

Nota: La figura muestra los resultados calculados del potencial energético.

Figura 4.

Resultados de las emisiones de $\mathrm{CO}_{2}$ a la atmósfera para las diferentes fuentes de biomasa.

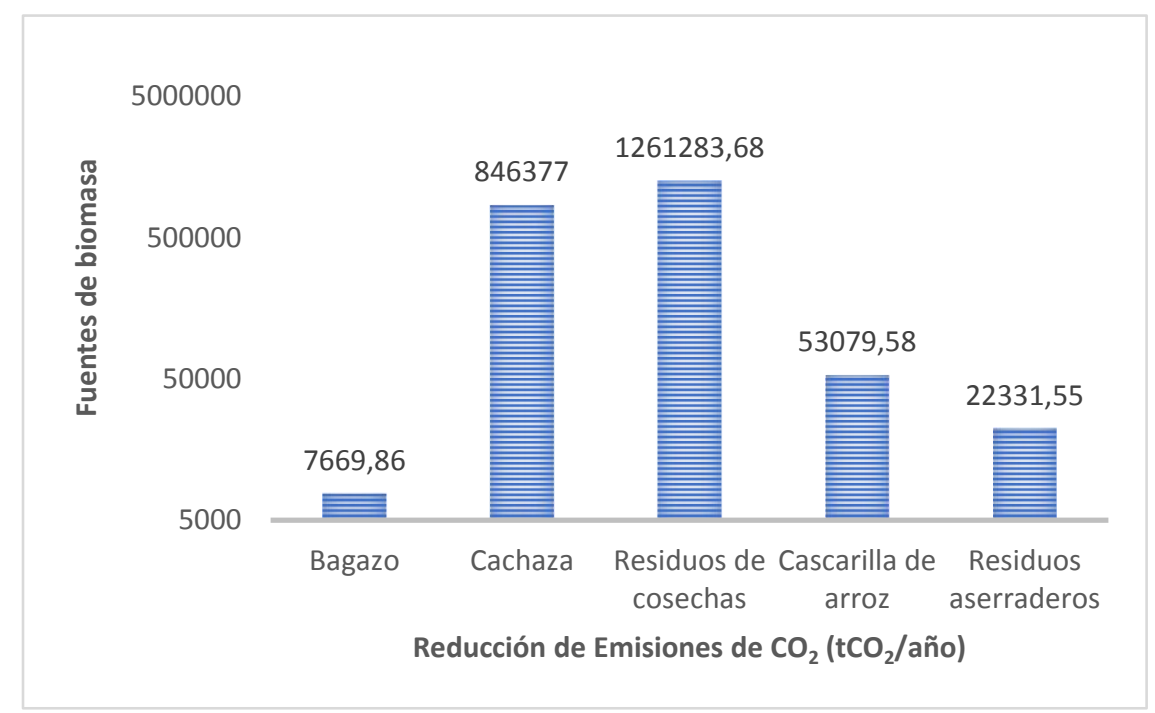

Nota: La figura resume la determinación de la reducción de emisiones de $\mathrm{CO}_{2}$ a la atmósfera por fuente de biomasas. 
En la figura 4 se aprecia que la mayor reducción de emisiones de $\mathrm{CO}_{2}$ la tienen los residuos de las cosechas, que alcanzan las 1.261.283,68 $\mathrm{tCO}_{2} /$ año.

Se puede concluir, luego de los resultados presentados anteriormente, que el bagazo es la biomasa más atractiva para ser utilizada como fuente de combustible. Aunque no produce las mayores reducciones de emisiones netas a la atmósfera, presenta los mayores aportes energéticos. Esto se sustenta fundamentalmente porque es la biomasa de mayor disponibilidad luego del levantamiento realizado.

\section{Desarrollo del modelo AHP}

Este método parte de una estructura jerárquica multinivel, que relaciona las alternativas modificadas, los subcriterios y los criterios, distribuyéndolos de acuerdo con la figura 5 .

\section{Figura 5.}

Estructura jerárquica.

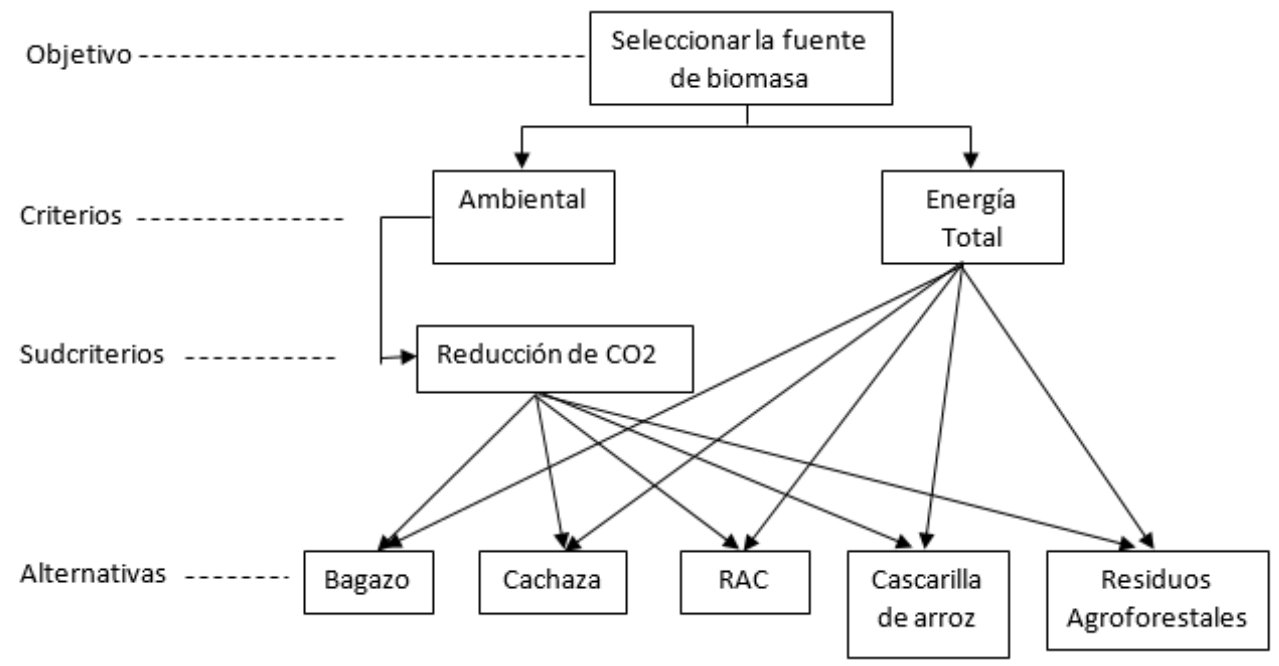

Fuente: elaboración propia.

Por otra parte, la comparación matricial por pares de las alternativas con respecto al subcriterio Reducción de $\mathrm{CO}_{2}$ se presenta en la tabla 4. Se traduce que el mayor peso o vector de prioridad de las alternativas en relación con el subcriterio de reducción de $\mathrm{CO}_{2}$ emitido a la atmósfera fue para los residuos forestales con un vector de prioridad (PVE en inglés) de 0,51, seguido de la cascarilla de arroz con un PVE de 0,273.

Desde el criterio de energía total sucede algo diferente. El bagazo presenta el mayor peso de prioridades en relación con las restantes alternativas evaluadas. Esta muestra un PVE de 0,576, seguido de los RAC con un PVE de 0,191. Es decir, estas dos biomasas 
Jiménez Borges, Álvarez González, López Bastida y Bermúdez Chou

se desempeñan mejor que las restantes, desde el punto de vista de energía total disponible.

\section{Tabla 4.}

Comparación matricial por pares de las alternativas con respecto al subcriterio reducción de $\mathrm{CO}_{2}$

\begin{tabular}{|c|c|c|c|c|c|c|}
\hline Alternativa & Bagazo & Cachaza & RAC & $\begin{array}{c}\text { Cascarilla de } \\
\text { arroz }\end{array}$ & $\begin{array}{c}\text { Residuos } \\
\text { forestales }\end{array}$ & $\begin{array}{c}\text { Vector de } \\
\text { prioridad (PVE) }\end{array}$ \\
\hline Bagazo & 1 & 0,25 & 0,333 & 0,143 & 0,111 & 0,036 \\
\hline Cachaza & 4 & 1 & 2 & 0,25 & 0,167 & 0,106 \\
\hline RAC & 3 & 0,5 & 1 & 0,2 & 0,143 & 0,072 \\
\hline $\begin{array}{c}\text { Cascarilla } \\
\text { de arroz }\end{array}$ & 7 & 4 & 5 & 1 & 0,333 & 0,273 \\
\hline $\begin{array}{c}\text { Residuos } \\
\text { forestales }\end{array}$ & 9 & 6 & 7 & 3 & 1 & 0,513 \\
\hline
\end{tabular}

Fuente: elaboración propia.

La tabla 5 resume la comparación matricial por pares de las alternativas respecto al criterio de energía total.

Tabla 5.

Comparación matricial por pares de las alternativas con respecto al criterio de energía total.

\begin{tabular}{|c|c|c|c|c|c|c|}
\hline Alternativa & Bagazo & Cachaza & RAC & $\begin{array}{c}\text { Cascarilla } \\
\text { de arroz }\end{array}$ & $\begin{array}{c}\text { Residuos } \\
\text { forestales }\end{array}$ & $\begin{array}{c}\text { Vector de } \\
\text { prioridad (PVE) }\end{array}$ \\
\hline Bagazo & 1 & 6 & 5 & 8 & 9 & 0,576 \\
\hline Cachaza & 0,167 & 1 & 0,5 & 3 & 4 & 0,130 \\
\hline RAC & 0,2 & 2 & 1 & 4 & 5 & 0,191 \\
\hline $\begin{array}{c}\text { Cascarilla de } \\
\text { arroz }\end{array}$ & 0,125 & 0,33 & 0,25 & 1 & 2 & 0,060 \\
\hline $\begin{array}{c}\text { Residuos } \\
\text { forestales }\end{array}$ & 0,111 & 0,25 & 0,2 & 0,5 & 1 & 0,040 \\
\hline
\end{tabular}

Fuente: elaboración propia. 
La figura 6 establece los pesos locales de las alternativas bajo cada criterio de decisión. Se evidencia que los residuos forestales — desde el punto de vista de reducción de CO2 - presentan el mayor peso local (38,51 \%), seguidos de la cascarilla de arroz $(22,14 \%)$. El bagazo obtiene su mayor peso de acuerdo con el criterio de energía total $(14,41 \%)$.

\section{Figura 6.}

Pesos locales de las alternativas bajo cada criterio de decisión

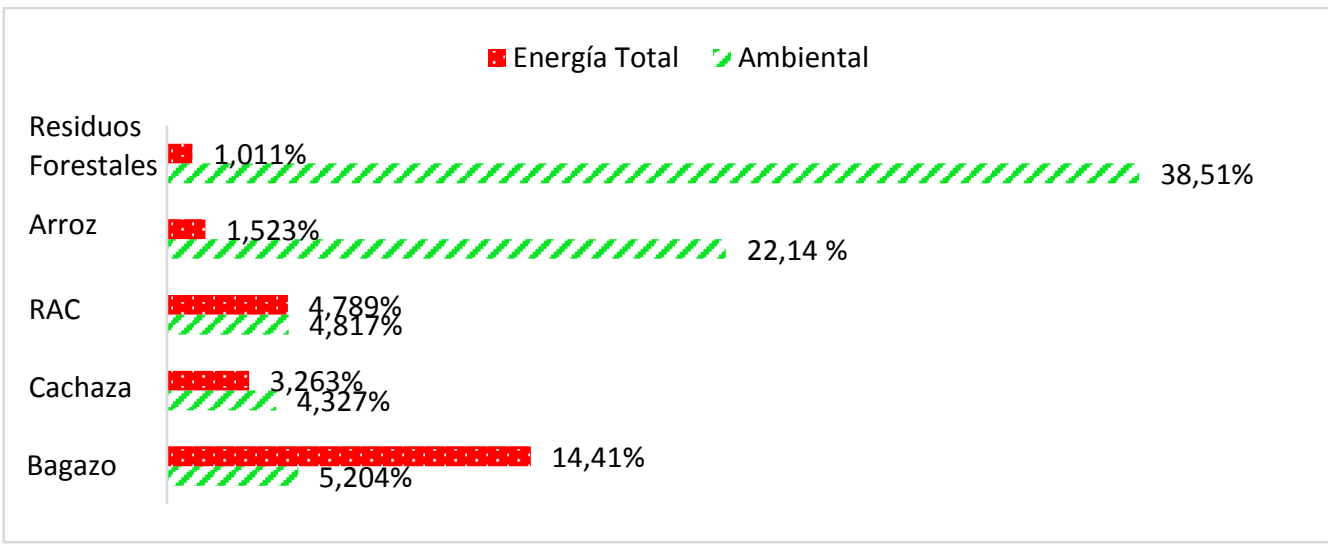

Fuente: elaboración propia.

\section{Análisis de sensibilidad}

Mediante el análisis de sensibilidad se puede constatar cómo varían las clasificaciones generales de las alternativas para la selección de la fuente de biomasa respecto a cambios en las prioridades de los criterios o subcriterios. Este análisis es muy utilizado en estudios que incluyen evaluación subjetiva para validar la solidez de los resultados (Al Garni et al., 2016).

Considerando un escenario de igual peso (50 \% para cada criterio), los resultados muestran que, en este caso, el bagazo presenta el mayor peso (32,3\%), seguido de los residuos agroforestales $(27,7 \%)$. Es decir, en un escenario de pesos iguales a los criterios de energía total y reducción de $\mathrm{CO}_{2}$, el bagazo es la biomasa de mayor sostenibilidad general.

\section{CONCLUSIONES}

Entre las diferentes fuentes de biomasa, el bagazo presenta el mayor aporte energético con $2,45 \times 10^{10}$, dado que solo se utiliza como combustible y presenta una energía útil de $2,33 \times 10^{10} \mathrm{MJ}$. 
La mayor reducción de $\mathrm{CO}_{2}$ se produjo con los residuos de cosecha, que alcanzaron 1.261.283 tCO 2 /año. La biomasa de menor contribución fue el bagazo con 7. 669,86 $\mathrm{tCO}_{2}$.

Mediante la aplicación del método AHP fue posible determinar que los residuos forestales presentaron el mayor PVE con 0,51 entre todas las alternativas estudiadas con relación al criterio de reducción de $\mathrm{CO}_{2}$ emitido a la atmósfera. Sin embargo, desde el punto de vista del criterio de energía total, el bagazo con PVE de 0,57 fue el de mayor contribución energética.

Al aplicar un análisis de sensibilidad, considerando un escenario de igual peso, los resultados muestran que para el bagazo (32,3 \%) y los residuos agroforestales (27,7 \%) se presenta el mayor peso. Es decir, en un escenario similar para los criterios de energía total y reducción de $\mathrm{CO}_{2}$, el bagazo es la biomasa de mayor sostenibilidad general.

\section{Referencias}

Ahmad, S., Tahar, R. M. (2014). Selection of renewable energy sources for sustainable development of electricity generation system using analytic hierarchy process: a case of Malaysia. Renewable Energy, 63, 458-466. doi: http://dx.doi.org/10.1016/j.renene.2013.10.001

Al Garni, H., Kassem, A., Awasthi, A., Komljenovic, D. y Al-Haddad, K. (2016). A multicriteria decision making approach for evaluating renewable power generation sources in Saudi Arabia. Sustainable Energy Technologies and Assessments,16,137-150. doi: http://dx.doi.org/10.1016/j.seta.2016.05.006

Amer, M. \& Daim, T. U. (2011). Selection of renewable energy technologies for a developing county: a case of Pakistan. Energy for Sustainable Development, 15, 420-435. doi: http://dx.doi.org/10.1016/j.esd.2011.09.001

Aras, H., Erdogmus, S. \& Koc, E. (2004). Multi-criteria selection for a wind observation station location using analytic hierarchy process. Renew Energy, 23, 13831392.

AZCUBA (2019). Grupo Empresarial de la Industria Azucarera. Información de las biomasas en el territorio.

Cerdá, E., Giuntoli et al. (2012). Energía obtenida a partir de biomasa. En Cuadernos Económicos de ICE (pág. 83).

Fajardo-Canales, G. E., Mejía-Pineda, N. y Gutiérrez-Bardales, J. (2018). Potencial calórico y acumulación de biomasa de la especie Leucaena macrophylla Benth. establecida con tres tipos de espaciamiento en Cortes, Honduras. Revista Forestal Mesoamericana Kurú, 15(Suppl. 1), 80-87. Epub July 19, 2019. doi: https://dx.doi.org/10.18845/rfmk.v15i1.3840

GAF (2019). Reporte de la situación de la biomasa arrocera y forestal. Grupo Agroforestal Cienfuegos. 
Giuntoli, J., Marelli, L., Lugato, E., Baxter, D., Agostini, A., y Caserini, S. (2016). Climate change impacts of power generation from residual biomass. Biomass and Bioenergy, 146-158.

doi: http://dx.doi.org/10.1016/j.biombioe.2016.02.024

Guerra, R. (2016). Cartelera de oportunidades de inversión extranjera 2016 - 2017.

Hernández, B. (2018). Oportunidades de inversiones en bioeléctricas. Paper presented at the 1st International Fair Energías Renovables Cuba, La Habana

Jiménez Borges, R, López Bastida, E.J, González Pérez, F, y Curbelo García J.A (2017). Metodología para la estimación del potencial de biomasa en Cienfuegos con fines energéticos. Revista de Investigaciones, 10(2).

Kazunobu Suzuki, N. T., Yoshihito Shirai., Mohd Ali Hassan., \& Mitsuru Osaki (2017). Evaluation of biomass energy potential towards achieving sustainability in biomass energy utilization in Sabah, Malaysia. Biomass and Bioenergy, 97, 149-154. doi: http://dx.doi.org/10.1016/j.biombioe.2016.12.023

Lee, A. H., Chen, H. H. \& Kang, H. Y. (2009). Multi-criteria decision making on strategic selection of wind farms. Renew Energy, 34, 120-126.

Munir Ozturka, N. S., Volkan Altay., Rizwan Iqbal., Khalid Rehman Hakeem., Mohammad Jawaid. \& Faridah Hanum Ibrahim. (2017). Biomass and bioenergy: An overview of the development potential in Turkey and Malaysia. Renewable and Sustainable Energy Reviews, 79, 1285-1302. doi: http://dx.doi.org/10.1016/j.rser.2017.05.111

Mustafa Ozcan , S. O. \& Yuksel Oguz. (2017). Potential evaluation of biomass-based energy sources for Turkey. Engineering Science and Technology, an International Journal, 1-7. doi: http://dx.doi.org/10.1016/j.jestch.2014.10.003

Narváez, R. P. (2013). Factor de emisión de CO2 debido a la generación de electricidad durante el periodo 2001-2011. ACI Avances en Ciencias e Ingenierías, 5(1).

Nixon, J. D., Dey, P. K. \& Gosh, S. K. (2013). Evaluation of options for energy recovery from municipal solid waste in India using the hierarchical analytical network process. Energy, 59, 215-223.

ONEI. (2019). Oficina Nacional de Estadística e Información. Anuario Provincial

Oscullo, J., y Haro, L. (2016). Factor anual de emisión de CO2 producido por el parque generador del Sistema Nacional Interconectado del Ecuador, mediante la aplicación de la metodología de la Convención Marco sobre el Cambio Climático UNFCCC, para el periodo 2009-2014. Revista Politécnica, 37(1), 61.

Ozoegwua, C. E., Onwosi, C.O., Mgbemene, C.A., \& Ozor, P.A. (2017). Biomass and bioenergy potential of cassava waste in Nigeria: Estimations based partly on rural-level garri processing case studies. Renewable and Sustainable Energy Reviews, 72, 625-638. doi: http://dx.doi.org/10.1016/j.rser.2017.01.031 
Qazi, W. A., Abushammala, M. F. M. \& Azam, M. H. (2018). Multi-criteria decision analysis of waste-to-energy technologies for municipal solid waste management in Sultanate of Oman. Waste Management \& Research, 36(7), 594-605. doi: https://doi.org/10.1177/0734242X18777800

Rahayu, D. E., Nasarani, D., Hadi, W. y Wrjodirjo, B. (2018). Potential of biomass residues from oil palm agroindustry in Indonesia. MATEC Web Conf., 197 13008. doi: https://doi.org/10.1051/matecconf/201819713008

Resolución conjunta de los Ministerios de Industria, E. y. (2016). Factores de emisión de $\mathrm{CO}_{2}$ y coeficientes de paso a energía primaria de diferentes fuentes de energía final consumidas en el sector de edificios en España.

Saaty, T. (1998). Método analítico jerárquico (AHP): principios básicos. En Evaluación y Decisión Multicriterio. Reflexiones y Experiencias (págs. 17-46). Universidad de Santiago.

Sanz, M. L. (2011). Optimización del aporte energético en nutrición artificial: Segunda lección Jesús Culebras. Nutrición Hospitalaria, 26(6).

Sheng Zhong, S. N., \& Yipeng Wang. (2018). Research on potential eEvaluation and sustainable development of rural biomass energy in Gansu Province of China. Sustainability, 10(2800), 2-20. doi: 10.3390/su10103800

Terence James, A., Beltrán Fundora, P., y Sendon Medina, A. (2010). Diseño de briqueteadora hidráulica para producir briquetas multitubulares de biomasas. Cienfuegos.

Toklu, E. (2017). Biomass energy potential and utilization in Turkey. Renewable Energy, 107, 235-244. doi: http://dx.doi.org/10.1016/j.renene.2017.02.008

Vanessa Burg, G. B., Matthias Erni., Renato Lemm., \& Oliver Thees. (2018). Analyzing the potential of domestic biomass resources for the energy transition in Switzerland. Biomass and Bioenergy, 111, 60-69. doi: https://doi.org/10.1016/j.biombioe.2018.02.007

Vergara Tamayo, C. A., y Ortiz Motta, D. C. (2016). Contribución al desarrollo sostenible local de los proyectos mdl en el sector de generación eléctrica por biomasa: INCAUCA SA CASE. Revista Facultad de Ciencias Económicas: Investigación y Reflexión, 24(2), 161-182.

Yiang Li, L. W. Z., \& Wang, R.Z. (2017). Urban biomass and methods of estimating municipal biomass resources. Renewable and Sustainable Energy Reviews, 80, 1017-1030. doi: http://dx.doi.org/10.1016/j.rser.2017.05.214

Yu Jiang, E. v. d. W., Ekko, C., van Ierland, Karel., \& Keesmana, J. (2017). The potential role of waste biomass in the future urban electricity system. Biomass and Bioenergy, 107, 182-190.

doi: http://dx.doi.org/10.1016/j.biombioe.2017.10.001 